Z Herz- Thorax- Gefäßchir 2017 · 31:20-22 DOI 10.1007/s00398-016-0118-x Online publiziert: 26 . Oktober 2016 (c) Springer-Verlag Berlin Heidelberg 2016

CrossMark

\section{Hintergrund}

Thorakale Aortenaneurysmen treten mit einer Häufigkeit von 4,2 \% in der Bevölkerung auf. Die Ätiologie der Aortenaneurysmen ist sehr vielfältig und reicht von degenerativen Veränderungen über hypertoniebedingte Dilatation bis zu seltenen genetischen Erkrankungen. Wie der Großteil der Aneurysmen sind Aneurysmen im Bereich der Aorta ascendens häufig asymptomatisch und werden meist als Zufallsbefund bei einer bildgebenden Diagnostik gefunden. Nur in seltenen Fällen treten Symptome wie Dysphagie, Heiserkeit, Brustschmerzen oder Zeichen der Aortenklappeninsuffizienz auf. Die schwerwiegenden Komplikationen von unbehandelten oder nichtdiagnostizierten Aneurysmen sind Ruptur und Dissektion. Der maximale Aortendurchmesser ist der wichtigste Risikofaktor für das Auftreten dieser Komplikationen. Weiterhin spielen eine familiäre Belastung sowie veränderte mechanische Eigenschaften der Gefäßwand bei Vorhandensein einer bikuspiden Aortenklappe und Bindegewebserkrankungen wie z. B. das Marfan-Syndrom eine Rolle.

Als konservative Therapieoptionen stehen lediglich die konsequente Blutdruckeinstellung und der Verzicht auf körperliche Anstrengung zur Vermeidung von Blutdruckspitzen zur Verfügung. Bei Patienten mit Marfan-Syndrom haben medikamentöse Therapieansätze mit $\beta$-Rezeptoren-Blockern oder Angiotensin-(AT)-II-Antagonisten, die zu einer Verzögerung der Progression des Aneurysmawachstums führen sollten, nicht $\mathrm{zu}$ dem gewünschten Erfolg geführt [5].

\title{
D. Aicher
}

Klinik für Thorax- und Herz-Gefäßchirurgie, Sektion Klappenchirurgie, Universitätskliniken des Saarlandes, Homburg, Deutschland

\section{Ersatz der Aorta ascendens}

\section{Wann und warum?}

Letztendlich erscheint nur die chirurgische Resektion des Aneurysmas sinnvoll. Die Entscheidung zum Ersatz der Aorta ascendens beinhaltet jedoch ein sorgfältiges Abwägen der spontanen Prognose der Erkrankung und des operativen Risikos.

\section{Spontanprognose}

\section{Allgemein}

Die Wachstumsrate thorakaler Aneurysmen beträgt $0,07-0,1 \mathrm{~cm} / \mathrm{Jahr}$ und nimmt mit der Größe des Aneurysmas zu [3]. Die jährliche Rate von Aortenkomplikationen steigt bei einem Durchmesser ab $5 \mathrm{~cm}$ deutlich an [3]. Das Risiko einer Ruptur oder Dissektion beträgt 6,5 \% bei einer Aneurysmagröße von 5,0 bis $5,9 \mathrm{~cm}$ und steigt auf $14,1 \%$ pro Jahr für Aneurysmen $\geq 6,0 \mathrm{~cm}$. Noch deutlicher wird der Unterschied, wenn man sich das proportionale Risiko anschaut. Das Rupturrisiko ist bei einer Aneurysmagröße von 5,0 bis $5,9 \mathrm{~cm} 11$-fach und bei Aneurysmen von mehr als $6,0 \mathrm{~cm} 25$ fach erhöht gegenüber Patienten mit Aneurysmen zwischen $4,0 \mathrm{~cm}$ und $4,9 \mathrm{~cm}$. Kontrovers diskutiert wird die Frage, ob zur Abschätzung des Rupturrisikos die Größe des Aneurysmas zur Körpergröße ins Verhältnis gesetzt werden soll.

\section{Bikuspide Aortenklappe und Marfan-Syndrom}

Die bikuspid angelegte Aortenklappe ist mit 1-2\% in der Bevölkerung der häufigste angeborene Herzfehler. Bei $60 \%$ der Patienten findet sich zusätzlich eine Dilatation der Aorta ascendens, deren Ursache bisher noch nicht geklärt ist. Ei- nerseits werden Strömungsturbulenzen des Blutes verantwortlich gemacht, die den Untergang von vaskulären Endothelzellen bewirken, was zu Apoptose und Atherosklerose der Aortenwand mit nachfolgender Dilatation führt. Auf der anderen Seite gibt es eine wachsende Zahl von Hinweisen, die einen gemeinsamen Pathomechanismus der Fehlanlage der Klappe und der damit verbundenen Aortopathie nahelegen. Dafür spricht ebenfalls, dass bei Patienten mit bikuspider Aortenklappe auch nach Ersatz der Aortenklappe eine Dilatation der Aorta ascendens auftreten kann - im Gegensatz zu Patienten mit trikuspider Aortenklappe, bei denen eine Verringerung des Aortendurchmessers nach Klappenersatz beschrieben wurde [12].

Bei Patienten mit bikuspider Aortenklappe [9] wurde ähnlich wie bei Patienten mit Marfan-Syndrom [7] ein schnelleres Fortschreiten der Erkrankung beobachtet (jährliche Wachstumsrate etwa $2 \mathrm{~mm}$ ). Das Marfan-Syndrom wird autosomal-dominant vererbt und führt $\mathrm{zu}$ Mutationen im FBN1-Gen, das Fibrillin1 codiert. Dadurch kommt es zu einer Schwächung der Gefäßwand.

Die phänotypischen Expressionen der Aortendilatation sind unterschiedlich. Während Patienten mit MarfanSyndrom häufig eine Dilatation der Aortenwurzel zeigen, beginnt das Aneurysma bei Patienten mit bikuspider Aortenklappe oft erst am sinutubulären Übergang. Bei beiden Erkrankungen wurde ein 8- bis 9-fach erhöhtes Risiko für Rupturen und Dissektionen, verglichen mit der Normalbevölkerung, beobachtet. 


\section{Risiko der chirurgischen Therapie}

Das Risiko der chirurgischen Therapie mit Ersatz der Aorta ascendens beträgt in mehreren Serien bei elektiven Eingriffen 3-5\% [1, 11]. Der zusätzliche Ersatz der Aortenwurzel mit Rekonstruktion [2] oder der Ersatz der Aortenklappe ist mit vergleichbarem Risiko durchführbar [4]. Durch elektiven Ersatz der Aorta ascendens wird die normale Lebenserwartung wiederhergestellt [1]. Dagegen steigt das Risiko des Aszendensersatzes unter Notfallbedingungen etwa um das 5-Fache an $(15,4-17,1 \%$; $[1,11])$.

Die klinische Erfahrung zeigt jedoch, dass das operative Risiko nicht einheitlich ist, sondern von Alter und Komorbiditäten des Patienten abhängt. Bei älteren Patienten ( $>70$ Jahre) mit chronischer Niereninsuffizienz ist die Krankenhaussterblichkeit mit 17,9\% hoch, was bedeutet, dass diese Patientengruppe von der Anwendung der Standardkriterien nicht profitiert [8]. Bei einem Alter über 80 Jahren steigt das Letalitätsrisiko weiter an, weshalb ein prophylaktischer Aortenersatz für asymptomatische Aneurysmata in dieser Altersgruppe zu überdenken ist. Im Gegenteil dazu ist das Letalitätsrisiko bei jüngeren Patienten ( $<70$ Jahre) ohne vorherige Herzoperation mit 1,8 \% gering [8].

\section{Resümee}

Patienten mit symptomatischen Aneurysmen müssen unabhängig vom Aortendurchmesser operiert werden.

Bei symptomfreien Patienten empfiehlt sich unter Berücksichtigung der Spontanprognose sowie des operativen Risikos, entsprechend den europäischen [10] und den amerikanischen Richtlinien [6], ein prophylaktischer Ersatz der Aortenwurzel/Aorta ascendens, wenn der maximale Durchmesser $5,5 \mathrm{~cm}$ überschreitet. Für Patienten mit bikuspider Aortenklappe oder Marfan-Syndrom ist ein operatives Vorgehen ab einem Durchmesser von $5 \mathrm{~cm}$ gerechtfertigt. Für Patienten mit erhöhtem chirurgischem Risiko (Alter >70 Jahre, Niereninsuffizienz, Voroperation) muss die RisikoNutzen-Abwägung neu überdacht wer- den. Eine großzügigere Entscheidung für ein chirurgisches Vorgehen scheint dagegen bei jungen Patienten vertretbar.

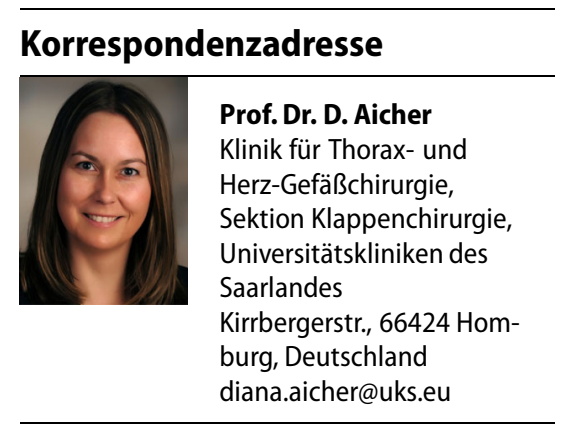

Interessenkonflikt. D. Aicher gibt an, dass kein Interessenkonflikt besteht.

\section{Literatur}

1. Achneck HE, Rizzo JA, Tranquilli M, Elefteriades JA (2007) Safety of thoracic aortic surgery in the present era. Ann Thorac Surg 84:1180-1185

2. Aicher D, Langer F, Lausberg $H$, Bierbach $B$, Schafers HJ (2007) Aortic root remodeling: tenyear experience with 274 patients. J Thorac Cardiovasc Surg 134:909-915

3. Davies RR, Goldstein LJ, Coady MA et al (2002) Yearly rupture or dissection rates for thoracic aortic aneurysms: simple prediction based on size. Ann Thorac Surg 73:17-27

4. Etz CD, Bischoff MS, Bodian C et al (2010) The Bentall procedure: Is it the gold standard? A series of 597 consecutive cases. J Thorac Cardiovasc Surg 140:S64-S70

5. Forteza A, Evangelista A, Sanchez V et al (2016) Efficacy of losartan vs. atenolol for the prevention of aortic dilation in Marfan syndrome: a randomized clinical trial. Eur Heart J 37:978-985

6. Hiratzka LF, Bakris GL, Beckman JA et al (2010) 2010 ACCF/AHA/AATS/ACR/ASA/SCA/ SCAI/SIR/STS/SVM guidelines for the diagnosis and management of patients with thoracic aortic disease: executive summary. A report of the American College of Cardiology Foundation/American Heart Association Task Force on Practice Guidelines, American Association for Thoracic Surgery, American College of Radiology, American Stroke Association, Society of Cardiovascular Anesthesiologists, Society for Cardiovascular Angiography and Interventions, Society of Interventional Radiology, Society of Thoracic Surgeons, and Society for Vascular Medicine. Catheter Cardiovasc Interv 76:E43-E86

7. Jondeau G, Detaint D, Tubach F et al (2012) Aortic event rate in the Marfan population: a cohortstudy. Circulation 125:226-232

8. Kunihara T, Aicher D, Asano M et al (2014) Risk factors for prophylactic proximal aortic replacement in the current era. Clin Res Cardiol 103:431-440

9. Michelena HI, Khanna AD, Mahoney D et al (2011) Incidence of aortic complications in patients with bicuspid aortic valves. JAMA 306:1104-1112
10. Vahanian A, Alfieri O, Andreotti F et al (2012) Guidelines on the management of valvular heart disease (version 2012): the Joint Task Force on the Management of Valvular Heart Disease of the European Society of Cardiology (ESC) and the European Association for Cardio-Thoracic Surgery (EACTS). Eur J Cardiothorac Surg 42:1-44

11. Williams JB, Peterson ED, Zhao Y et al (2012) Contemporary results for proximal aortic replacement in North America. J Am Coll Cardiol 60:1156-1162

12. Yasuda H, Nakatani S, Stugaard M et al (2003) Failure to prevent progressive dilation of ascending aorta by aortic valve replacement in patients with bicuspid aortic valve: comparison with tricuspid aortic valve. Circulation 108:II-291-II-294 
Hier steht eine Anzeige.

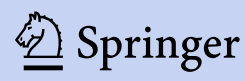

